\title{
Spontaneous Rupture of the Bladder after a Seizure: A Pitfall of Diagnosis
}

\author{
Eugene Lin ${ }^{1,2,3}$, Ho Lin ${ }^{2,4,5}$, Guang-Uei Hung ${ }^{6}$, Jungle Chi-Hsiang $\mathrm{Wu}^{1}$, Huei-Loong Hsieh ${ }^{7}$, \\ Tah-Chong Lin $^{3}$, Chao-Chih Chen ${ }^{3}$, Chien-Hsiang Wang ${ }^{3}$, Mao-Sheng Lin ${ }^{3 *}$ \\ ${ }^{1}$ Department of Urology, Chang Bing Show Chwan Memorial Hospital, Changhua, Chinese Taipei \\ ${ }^{2}$ Department of Life Sciences, National Chung Hsing University, Taichung, Chinese Taipei \\ ${ }^{3}$ Department of Urology, Show Chwan Memorial Hospital, Changhua, Chinese Taipei \\ ${ }^{4}$ Graduate Institute of Rehabilitation Science, China Medical University, Taichung, Chinese Taipei \\ ${ }^{5}$ Department of Urology, University of Texas Southwestern Medical Center, Dallas, USA \\ ${ }^{6}$ Department of Nuclear Medicine, Chang Bing Show Chwan Memorial Hospital, Changhua, Chinese Taipei \\ ${ }^{7}$ Department of Urology, Chushang Show Chwan Hospital, Nantou City, Chinese Taipei \\ Email: ${ }^{*}$ s0924.tw@yahoo.com.tw
}

Received December 5, 2012; revised January 7, 2013; accepted January 16, 2013

\begin{abstract}
Seizures can lead to different injuries ranging from minor lacerations to serious head injuries or bony fractures. We report a rare case of a male patient presenting with a history of a distended abdomen and gross hematuria following a seizure attack. After catheterization, the drained urine was bloodstained. On clinical suspicion of an acute abdomen, computed tomography was conducted, which showed intraperitoneal rupture of the bladder. At laparotomy, we found and repaired a laceration in the dome of the bladder in 3 layers. The postoperative period was uneventful. Our case shows that a complete abdominal examination is needed on patients following a generalized seizure episode. We also suggest that certain drugs for treating schizophrenia have anticholinergic effects, which may induce chronic urine retention. Chronic urine retention, especially in elderly male patients, may contribute to the risk of a spontaneous rupture of the bladder.
\end{abstract}

Keywords: Bladder Injury; Provoked Seizure; Intraperitoneal Bladder Rupture; Seizure

\section{Introduction}

Patients with epilepsy disease occasionally suffer injuries during a seizure. Most of these injuries occur during generalized myoclonic seizures or tonic clonic seizures [1]. Although many types of injuries have been described, a bladder injury is extremely rare [2].

A patient presented with a distended abdomen after a generalized tonic clonic seizure. An abdominal ultrasonography revealed ascites over the lower abdomen and a distended bowel loop. After inserting a foley, we also noted gross hematuria. On clinical suspicion of an acute abdomen, we used computed tomography (CT) and found an intraperitoneal rupture of the bladder.

Under the impression of the intraperitoneal rupture of the urinary bladder, we performed a laparotomy to repair the bladder. We reviewed the literature and discussed the possible mechanisms of a bladder rupture caused by seizures.

"Corresponding author.

\section{Case Report}

Our case study was of a 60 -year-old man with a 15-year medical history of schizophrenia and mood disorders. When he was young he was an alcoholic. He was admitted to our psychiatric chronic ward for 3 years, during which he took Olanzapine $5 \mathrm{mg}$ HS, Estazolam $2 \mathrm{mg} \mathrm{HS}$, and Biperiden HCL $2 \mathrm{mg}$ TID daily to treat his schizophrenia. For a long time this patient had lower urinary symptoms such as urinary hesitancy and a small stream, but he did not receive any medical treatment. Early one morning he suddenly had a seizure and lost consciousness. This general seizure lasted $1 \mathrm{~min}$. Thereafter, he was transferred to our medical intensive care unit for further evaluation and treatment. According to the psychiatric ward care nurses, he did not have a fever, chills, hemiplegia, or a history of epilepsy prior to this general seizure attack. A CT scan of his brain showed no visible cerebral or cerebellar parechymal abnormal density such as a hemorrhage or an infarction. A check of the surveillance video recording showed that this patient did not 
suffer from any violent episodes.

He had no history of hematuria or of any urological disease. When he was transferred to our unit, he was conscious and alert. In our physical examination he had no pallor. His pulse rate was 82 per minute, and his blood pressure was 114/76 mmHg. His abdomen was distended, and we noted a generalized tenderness with suprapubic dullness extending up to the umbilicus. He had decreased bowel sounds. Because he had not passed any urine for more than 8 hours, we inserted a catheter and drained $300 \mathrm{~mL}$ of bloodstained urine with some blood clots. His abdomen was still distended after catheterization, and signs of peritonitis still persisted. After the catheterization, we performed an ultrasonography, which showed free fluid in the abdomen, but the other viscera were normal. Ultrasound-guided abdominal tapping revealed some ascites over the lower abdomen and a distended bowel loop. We performed ultrasound-guided abdominal tapping. The ascitic fluid was bloody and contained RBC $70,000 \mu \mathrm{L}$ (reference range is $<2000 / \mu \mathrm{L}$ ), WBC $2050 \mu \mathrm{L}$ of which $90 \%$ were neutrophils (reference range is < $300 / \mu \mathrm{L}$, neutrophils $18 \%$ - $46 \%$ ); the smear was negative for Gram's stain and acid fast stain, and we found no malignant cells (Table 1). On clinical suspicion of an acute abdomen, we performed a CT scan, which showed intraperitoneal rupture of the bladder (Figure 1). His serum white count was elevated. His biochemical parameters such as the liver and renal functions were within reference limits, except for his lower serum sodium level being $111 \mathrm{mmol} / \mathrm{L}$ (reference range is 135 - $147 \mathrm{mmol} / \mathrm{L}$ ). The serum lactic acid level was $99.9 \mathrm{mg} / \mathrm{dL}$ (reference range is 4.5 - $19.8 \mathrm{mg} / \mathrm{dL}$ ) (Table 1). We performed an emergency laparotomy under general anesthesia and repaired in 3 layers a $5 \mathrm{~cm}$ laceration wound present over the dome of the bladder. We also performed a suprapubic cystostomy. His postoperative recovery was uneventful. We added an alpha-blocker as Alfuzosin $10 \mathrm{mg}$ HS to treat his lower urinary symptoms. We also administered $25 \mathrm{mg}$ of Urecholine 3 times daily to release the chronic urine retention problem. Because he had the underlying disease of schizophrenia, he could not care for himself. His cystostomy catheter was removed 2 weeks post-operation, and the foley catheter was removed 3 weeks after the postoperative day, following which he voided normally. We did not administer anymore drugs to treat or prevent seizures or epilepsy after the operation. This patient was still admitted to our psychiatric chronic ward.

\section{Discussion}

Seizures always lead to various injuries ranging from head injuries, tongue biting, dental injuries, facial injuries, and even bony fractures. The risk of major trauma is higher in generalized tonic clonic seizures and myoclonic
Table 1. Laboratory findings of the patient.

\begin{tabular}{ccc}
\hline Variable & Patient's data & Normal range \\
\hline Urine & & \\
RBC (/HPF) & numerous & $0-5$ \\
WBC (/HPF) & $5-10$ & $0-5$ \\
Ascites tapping fluid & & \\
RBC ( $\mu \mathrm{L})$ & 70,000 & $<2000$ \\
WBC ( $\mu \mathrm{L})$ & 2050 & $<300$ \\
Neutrophils $(\%)$ & 90 & $18-46$ \\
Serum & 10.2 & $14-18$ \\
Hb (g/dL) & 18,800 & $4500-10,000$ \\
WBC & 20 & $2-6$ \\
Band $(\%)$ & 111 & $135-147$ \\
Sodium (mmol/L) & 99.9 & $4.5-19.8$ \\
\hline Lactic acid (mg/dL) & &
\end{tabular}

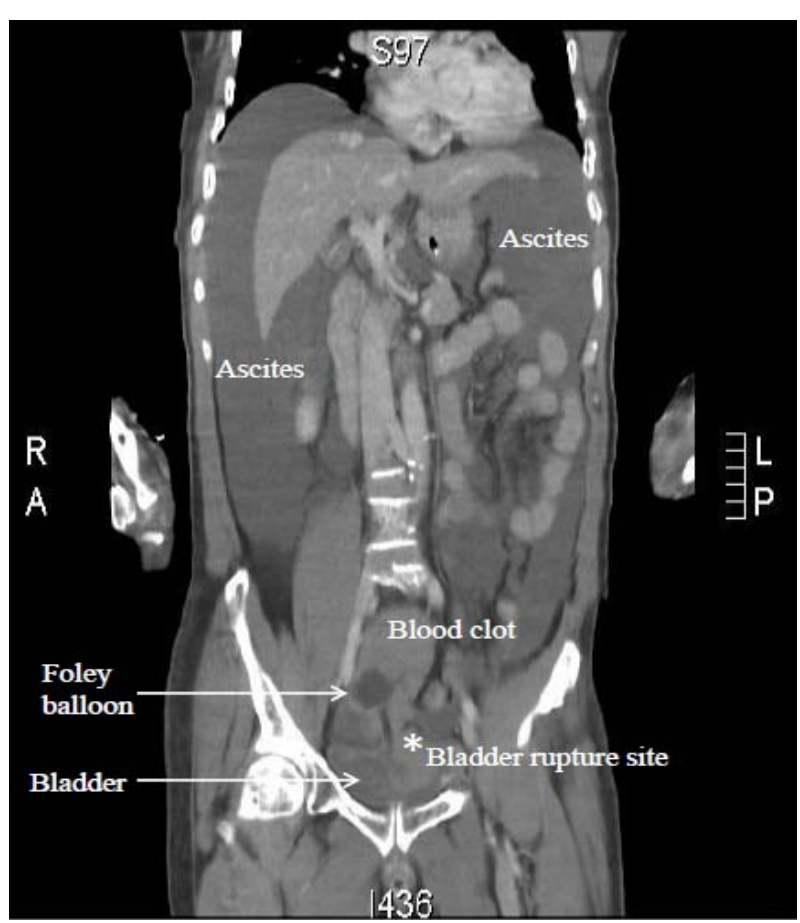

Figure 1. Computed tomography showed contrast in the peritoneal cavity suggesting intraperitoneal rupture of the bladder.

seizure attacks [1,3]. The seizure type, severity, and frequency are the main predictors of the seriousness of the injuries [4]. A spontaneous bladder rupture is an extremely rare case. After searching the Pubmed database, we found that to date only 5 cases of bladder injuries following a seizure attack have been reported. Pal et al. 
suggested that such an injury may be extremely rare because the bladder usually loses control and empties during a generalized seizure episode [2].

In our patient, seizure occurred in the early morning after he had retained urine overnight. For 3 years this patient had lower urinary symptoms such as urinary hesitancy and a small stream, but he did not receive any medical treatment. Lawn et al. proposed that the anticholinergic effects of the drug treatment for schizophrenia may have contributed to the risk of a spontaneous rupture of the bladder [1]. According to the pharmacy guide, one side effect of Biperiden HCL, a drug that was administered to this patient, is urine retention. Moreover, schizophrenic patients have been reported to have detrusor hypereflexia. In older male patients, the combination of a full bladder with a possible outflow obstruction, and the presence of raised intra-abdominal pressure during a general seizure attack could result in a spontaneous ruptured bladder [3]. We suggest that in our case the bladder ruptured because of a sudden rise in intra-abdominal pressure and because the bladder was fully distended from overnight retention. The fully distended bladder was relatively thinned out, especially in the anatomic unsupported dome area [4]. This predisposed the bladder to rupture at this weak point with the sudden rise in intra-abdominal pressure induced by a seizure attack [2]. In earlier reported cases, the injury occurred by a similar mechanism, probably because of a sudden rise of intra-abdominal pressure in the background of a full bladder [2]. This was attributed to the patient being on antipsychotic drugs with anticholinergic properties. Another possible mechanism is of the bladder rupturing first, inducing a provoked seizure. Provoked seizures are single seizures that may occur because of trauma, low blood sodium, hypoglycemia, high fever, or alcohol or drug abuse [5]. According to the psychiatric medical record, this patient had suicidal ideation and episodes of violent behavior before his admission, but these psychiatric conditions were under control before his bladder rupture episode. Although we did not detect any violent episodes from the surveillance video recording or from our physical examination, we could not exclude the possibility that the patient could have hit himself and have induced the sudden rise in abdominal pressure. A ruptured bladder may have caused the low serum sodium level and induced the provoked seizure.

$\mathrm{CT}$ is the most powerful diagnostic imaging tool for detecting a ruptured bladder. Chan et al. indicated that CT cystography is accurate for diagnosing bladder rupture. A coronal view, a sagittal view, and a multiplanar reconstruction may be useful in identifying most sites of bladder rupture. For an intraperitoneal rupture, the sensitivity and specificity were $100 \%$ and $99 \%$, respectively, in their database [6]. In recent years CT cystography has replaced conventional retrograded cystography in most trauma centers in diagnosing patients with suspected bladder rupture [6]. Shin et al. indicated that the sentinel clot sign on the bladder dome resulted in a high possibility of intraperitoneal bladder rupture. The sentinel clot sign signifies a relative high attenuating (range 52 to 68 HU) blood clot that accumulates near the site of the injury [7]. The sentinel clot sign on the CT may help with the diagnosis of a bladder injury, especially when trauma patients present with gross hematuria.

Pal et al. indicated that physicians must exercise extra caution when evaluating patients after a seizure attack because the patient can easily forget the abdominal injury episode, leading to a delayed diagnosis or misdiagnosis and increased patient morbidity and mortality [2]. We also suggest that the physician perform a careful abdominal examination on a patient following a seizure episode.

\section{REFERENCES}

[1] N. D. Lawn, W. R. Bamlet, K. Radhakrishnan, P. C. O'Brien and E. L. So, "Injuries Due to Seizures in Persons with Epilepsy: A Population-Based Study,” Neurology, Vol. 63, No. 9, 2004, pp. 1565-1570. doi:10.1212/01.WNL.0000142991.14507.B5

[2] B. C. Pal, S. Kumar, L. N. Dorairajan and N. Khattar, "Generalized Seizure: A Rare Etiology of Intraperitoneal Rupture of the Urinary Bladder,” Indian Journal of Urology, Vol. 23, No. 4, 2007, pp. 467-468. doi:10.4103/0970-1591.36723

[3] A. D. Irving and A. M. Drayson, "Bladder Rupture during ECT,” British Journal of Psychiatry, Vol. 144, No. 6, 1984, p. 670.

[4] U. K. Misra, J. Kalita, A. Vajpeyee, A. Mandhani and R. K. Sharma, "Bladder Rupture: A Rare Complication of Tonic-Clonic Seizures,” QJM, Vol. 96, No. 7, 2003, pp. 542-543. doi:10.1093/ajmed/hcg081

[5] D. G. Kasteleijn-Nolst Trenite, "Provoked and Reflex Seizures: Surprising or Common?” Epilepsia, Vol. 53, No. S4, 2012, pp. 105-113. doi:10.1111/j.1528-1167.2012.03620.x

[6] D. P. Chan, H. H. Abujudeh, G. L. Cushing Jr. and R. A. Novelline, "CT Cystography with Multiplanar Reformation for Suspected Bladder Rupture: Experience in 234 Cases," American Journal of Roentgenology, Vol. 187, No. 5, 2006, pp. 1296-1302. doi:10.2214/AJR.05.0971

[7] S. S. Shin, Y. Y. Jeong, T. W. Chung, W. Yoon, H. K. Kang, T. W. Kang and H. Y. Shin, "The Sentinel Clot Sign: A Useful CT Finding for the Evaluation of Intraperitoneal Bladder Rupture Following Blunt Trauma," Korean Journal of Radiology, Vol. 8, No. 6, 2007, pp. 492-497. doi:10.3348/kjr.2007.8.6.492 